\title{
Use of Intracardiac Echocardiography for Congenital and Structural Heart Disease Interventions
}

\author{
Mike Saji, MD*, D. Scott Lim, MD \\ Division of Cardiovascular Medicine, Department of Medicine, Advanced Cardiac Valve Center, University of Virginia, Charlottesville, \\ Virginia, USA
}

\begin{abstract}
Intracardiac echocardiography (ICE) has been commonly utilized for intraprocedural monitoring during structural heart interventions, and AcuNav is the most widely used device. Its imaging quality has been dramatically improved with the addition of a phasedarray system in recent years, and it has been used as an alternative to transesophageal echocardiography (TEE), especially in cases of atrial septal defect and patent fossa ovale closure. One advantage of ICE over TEE is that it does not require general anesthesia with endotracheal intubation, which means it is less invasive procedure. However, its application during other structural interventions remains in an early stage due to technologic limitations. Multiplanar imaging and wide-sector three-dimensional imaging are required to improve its utility.

Copyright $\odot 2016$ Science International Corp.
\end{abstract}

\section{Key Words}

Intracardiac echocardiography • Phased-array system • Real-time imaging • Three-dimensional imaging • Advantage - Transesophageal echocardiography

\section{Clinical Use of Intracardiac Echocardiography}

Intracardiac echocardiography (ICE) has been commonly used as an intraprocedural modality during structural heart interventions. It provides real-time anatomical assessment, and in certain procedures it has been used as an alternative to transesophageal echocardiography (TEE). ICE imaging quality was dramatically improved with the recent addition of

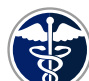

Fax +1 2037853346

E-Mail: jshd@scienceinternational.org

http://structuralheartdisease.org/ a phased-array system [1]. There are currently five types of commercially available ICE catheter systems: AcuNav (Siemens Medical Solutions, Malvern, PA, USA), UltralCE (Boston Scientific, Marlborough, MA, USA), EP Med View Flex (St. Jude Medical, St. Paul, MN, USA), ClearICE (St. Jude Medical), and SoundStar Catheter (Biosense Webstar Diamond Bar, CA, USA) [2]. AcuNav is currently the most widely utilized system during structural heart interventions. There are 8- and 11-French (F) catheters, and either the femoral or jugular vein is commonly used. It is capable of producing frequencies between 5 and $10 \mathrm{MHz}$, resulting in adequate imaging up to 10 to $14 \mathrm{~cm}$ from the transducer. This allows visualization of most left-sided structures from the right side of the heart. For selected interventions, ICE can be introduced into the left ventricle or atrium to produce additional images. The orientation marker on the screen shows the position of the handle such that inferior and superior structures are on the left and side of the screen, respectively. Fourway articulation of the catheter tip enables excellent maneuverability for optimal imaging, and full Doppler capabilities including color, tissue, and spectral Doppler provides additional information.

Compared with TEE, ICE provides many advantages. It does not require general anesthesia with endotracheal intubation and is therefore less invasive. Furthermore, fluoroscopy time, interventional procedural time, and hospital stay have are shortened [3], all of which could reduce total intervention cost.

Potential complications that may result from rightsided ICE imaging are extremely rare and include

* Corresponding author:

D. Scott Lim, MD

Advanced Cardiac Valve Center, Department of Medicine

University of Virginia

1215 Lee Street, Hospital Expansion, Room 4033, Charlottesville, VA, 22908, USA

Tel.: +1 434243 1146; Fax: +1 434244 7585; E-Mail: sl9pc@virginia.edu 
those associated with right heart catheterization such as pericardial tamponade, pulmonary embolism, infection, and bleeding.

\section{Structural Views}

Home View (Figure 1)

The ICE catheter is advanced into the right atrium under fluoroscopic guidance from the femoral vein. Since ICE does not have a wire lumen and uses a relatively stiff shaft, careful advance is necessary to avoid vascular complications. The probe in the neutral position within the right atrium allows for visualization of the right atrium, right ventricle, and tricuspid valve. It is often useful to return to this view when the operator is confronted with an unfamiliar view following multiple steering manipulations.

\section{Right Ventricular Outflow Tract View (Figure 2)}

From home view, clockwise rotation of approximately $30^{\circ}$ brings the right ventricular outflow tract (RVOT), pulmonary artery, and aortic valve into view.

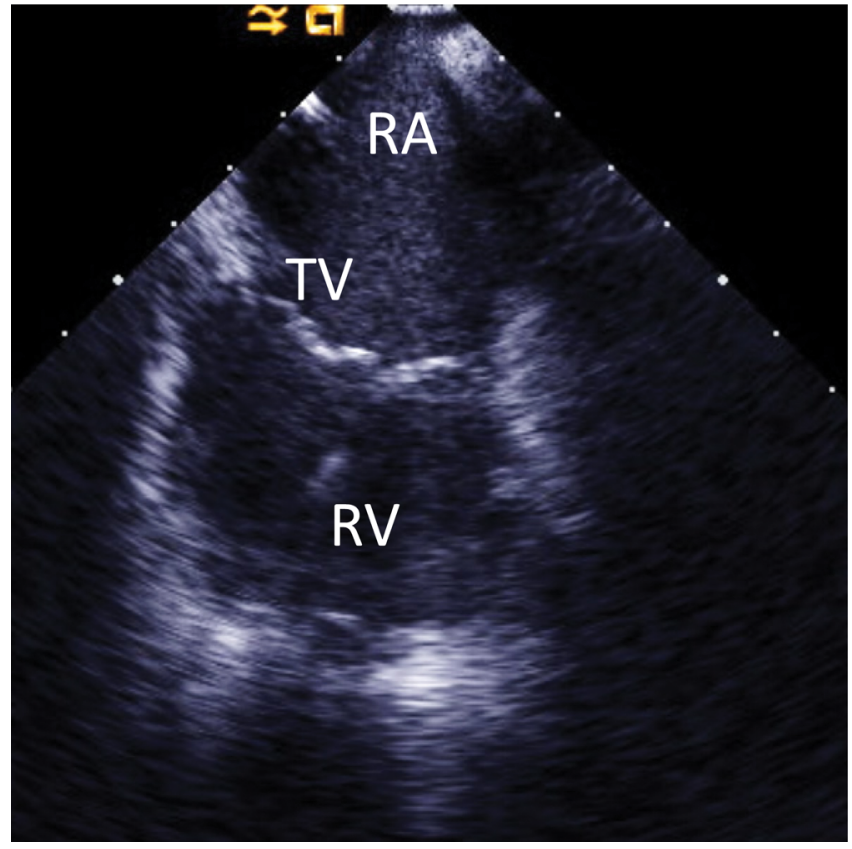

Figure 1. Home view. The catheter is advanced into mid RA with a neutral position. $\mathrm{RA}=$ right atrium; $\mathrm{RV}=$ right ventricle; $\mathrm{TV}=$ tricuspid valve.

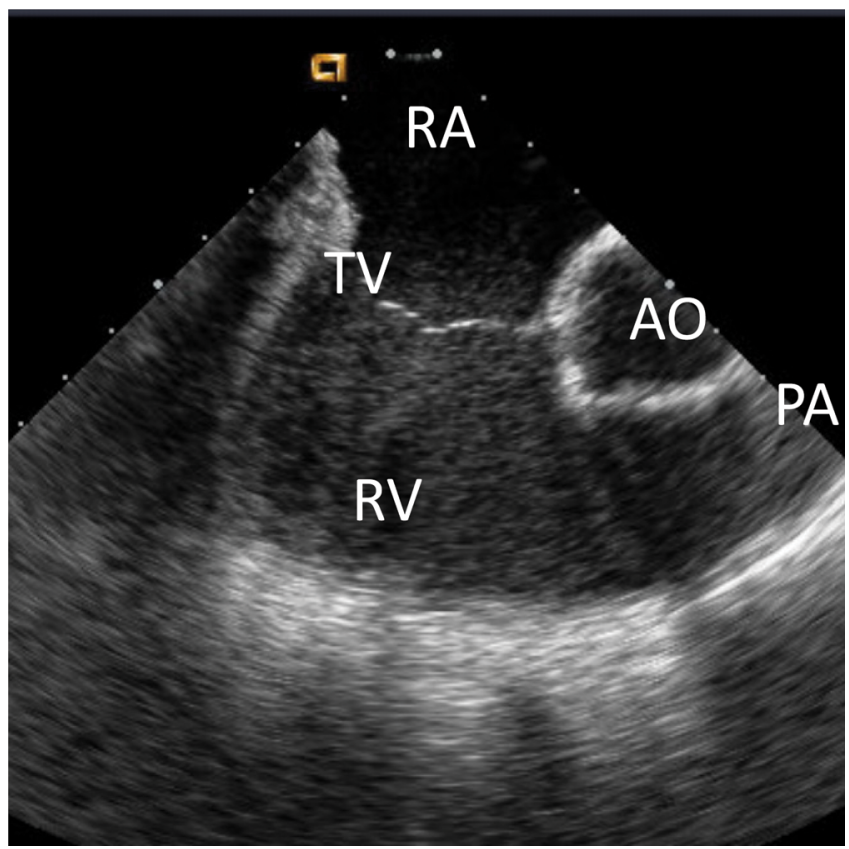

Figure 2. Right ventricle outflow tract view. The catheter is rotated clockwise with approximately $30^{\circ}$ from home view. $A O=$ ascending aorta; $\mathrm{PA}=$ pulmonary artery; $\mathrm{RA}=$ right atrium; $\mathrm{RV}=$ right ventricle; TV = tricuspid valve.

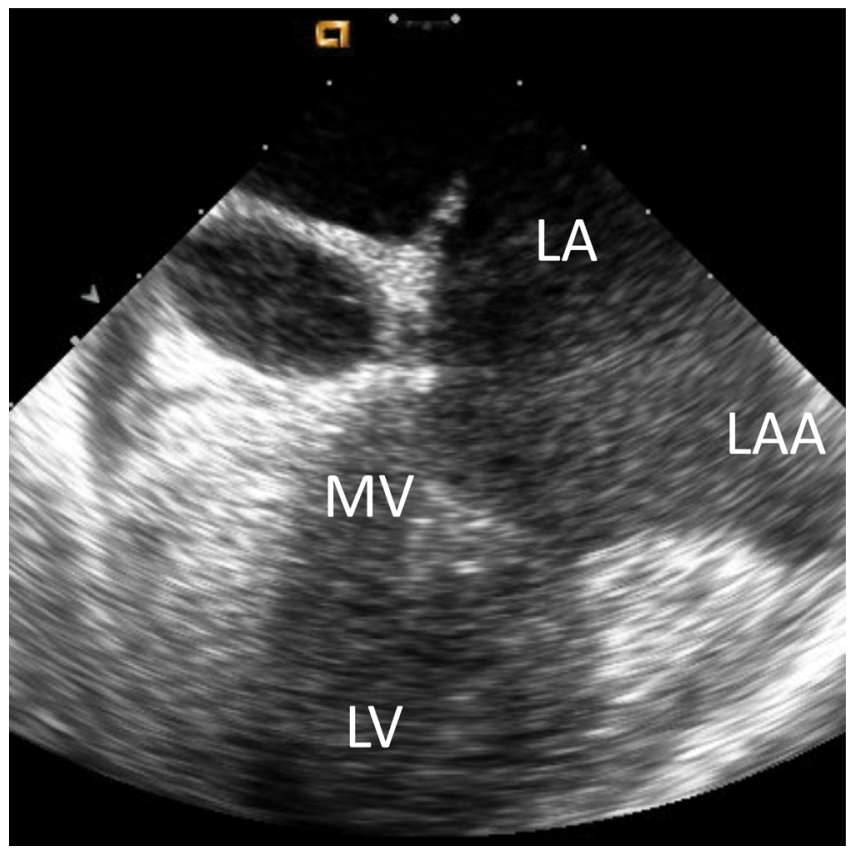

Figure 3. Left atrial appendage, mitral valve view. The catheter is rotated with approximately $60^{\circ}$ from home view. LA = left atrium; $\mathrm{LV}=$ left ventricle; $\mathrm{MV}=$ mitral valve. 


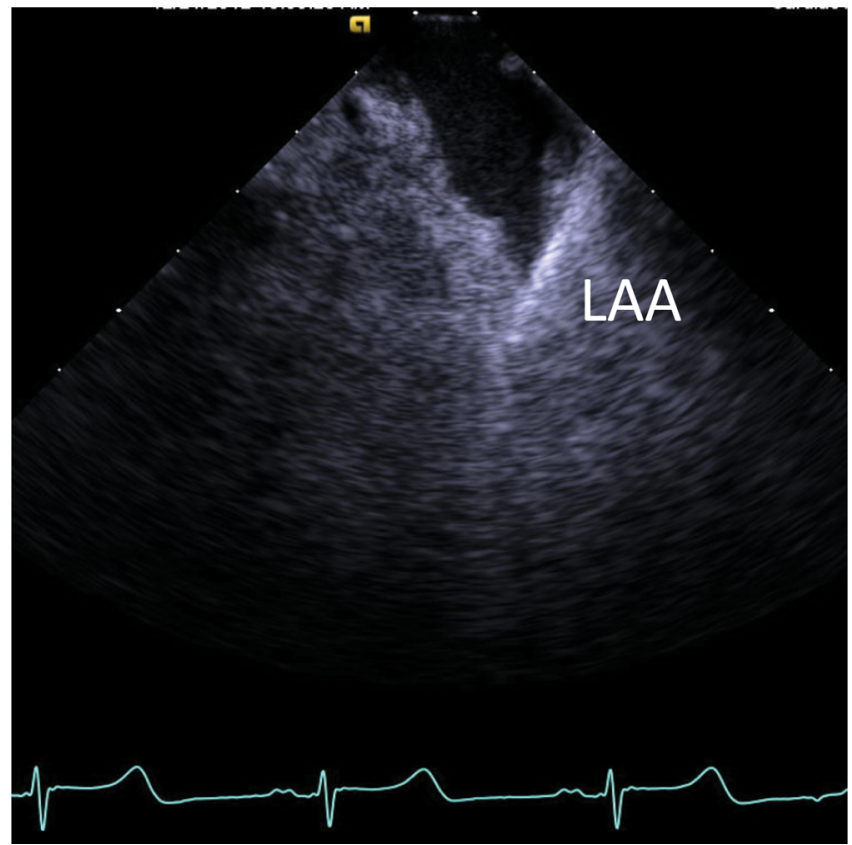

Figure 4. Image of the left atrial appendage (LAA) obtained from left atrium (LA) through the transseptal sheath. Intracardiac echocardiography advanced into the LA shows no thrombus in the LAA.

\section{Left Atrial Appendage, Mitral Valve View (Figure 3)}

Further rotation of the catheter approximately $60^{\circ}$ brings the mitral valve and the left atrial appendage. In selected patients, the left atrial appendage can be adequately evaluated from the right atrium to be sure it is clear of thrombus by two-dimensional ICE due to highly morphological variety of left atrial appendages. If there is not adequate visualization from the right side, the ICE catheter must be introduced into the coronary sinus [4], RVOT, left pulmonary artery [5], or left atrium [6] following transseptal puncture to confirm that the appendage is free of thrombus (Figure 4).

\section{Left Pulmonary Vein, Fossa Ovalis View (Long Axis View)} Figures 5 and 6

Another rotation of more than $90^{\circ}$ from the home view brings the left atrium and left pulmonary veins into view. Posterior tilt allows clear visualization of the fossa ovalis. The superior vena cava (SVC) can be seen by advancing the ICE catheter further to the superior right atrium, while the inferior vena cava (IVC) can be seen by

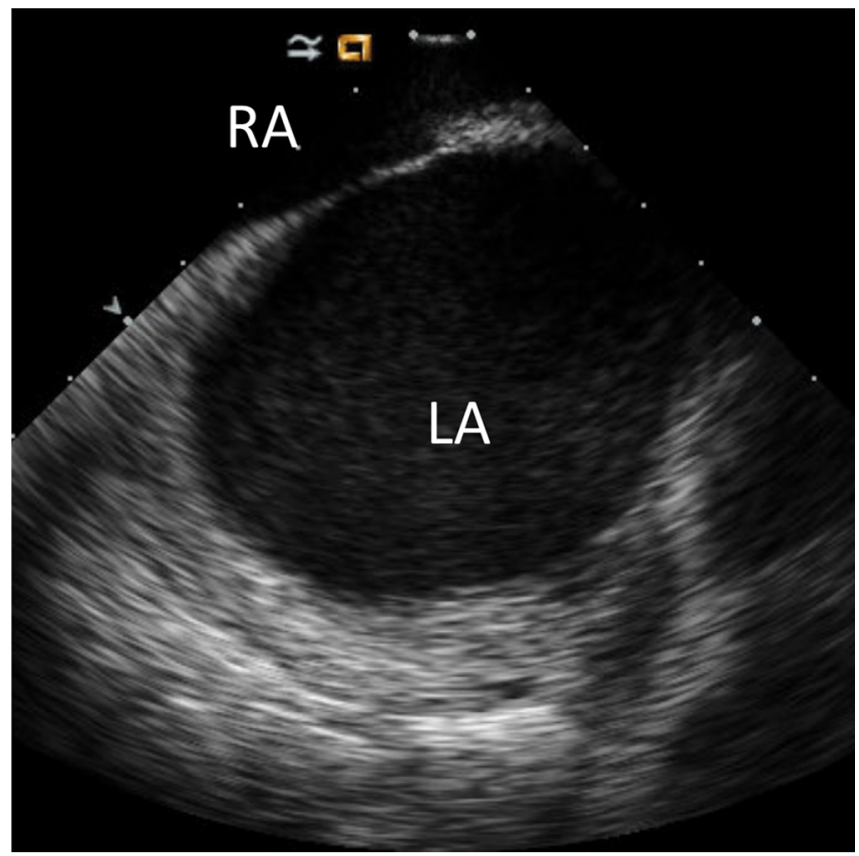

Figures 5 and 6. Left pulmonary vein, fossa ovalis view (long axis view of the septum). The catheter is rotated more than $90^{\circ}$ from home view. Posterior tilt allows visualization of the fossa ovalis. LA = left atrium; LSPV = left superior pulmonary vein; LIPV = left inferior pulmonary vein; $\mathrm{RA}=$ right atrium. 
retracting the ICE catheter to the inferior right atrium in this view. The superior rim, SVC rim, and IVC rim can be evaluated in this view in atrial septal defect (ASD) closure.

\section{Short Axis View (Figure 7)}

The ICE catheter is retracted to the inferior right atrium and tilted posteriorly and rightward. This brings the interatrial septum and aortic valve into view. This view is used to evaluate paravalvular leakage after transcatheter aortic valve replacement (TAVR), as well as measurement of aortic and posterior rims during $\mathrm{ASD}$ closure.

\section{Left Ventricular Long Axis View (Figure 8)}

The posterior-tilted catheter is rotated clockwise and then advanced into the right ventricle. The left ventricle can be imaged across the ventricular septum. Left ventricular function, mitral regurgitation, the subvalvular apparatus, and pericardial effusion can also be evaluated in this view. It may be of limited utility for interventions on the mitral valve and ventricular septum in this view.

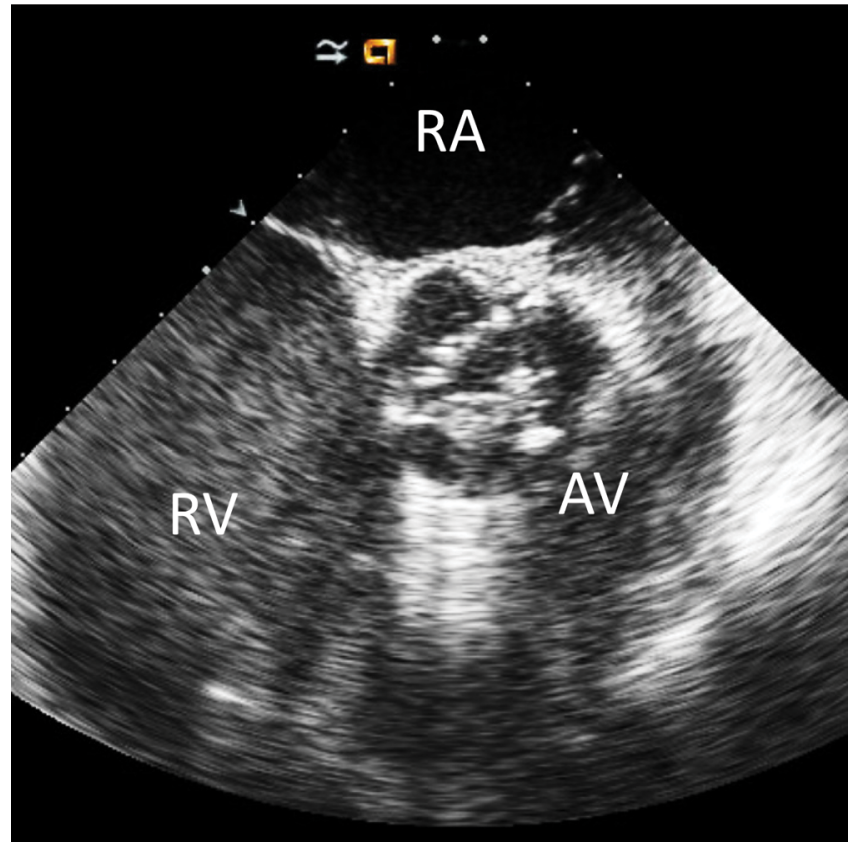

Figure 7. Short axis view. The catheter is retracted to the inferior right ventricle with a posterior tilt. $A V=$ aortic valve; $\mathrm{RA}=$ right atrium; RV = right ventricle.

\section{Specific Procedure Use}

Current clinical applications of ICE in cardiac interventional procedures include guidance of transseptal puncture [7], ASD/patent foramen ovale (PFO) closure [8, 9], percutaneous mitral balloon valvuloplasty [10], and TAVR [11]. It can be also used to guide diagnostic evaluation of cardiac mass [12], coronary sinus pacemaker lead placement [13], ventricular septal defect closure [14], transluminal septal ablation for hypertrophic cardiomyopathy [15], and potentially other structural heart interventions such as transcatheter mitral valve repair with the MitraClip and left atrial appendage closure.

\section{Guidance of Transseptal Puncture}

ICE enables safe navigation of transseptal puncture compared with traditional fluoroscopy guidance, particularly if patients have an unusual anatomical abnormality [7] (Figure 9). It may also help to reduce and immediately identify complications related to transseptal puncture. Optimal septum imaging is obtained by the long axis view such that the interatrial septum is perpendicular to the ultrasound beam. Tenting of the septum indicates stable contact of the needle or dilator with the

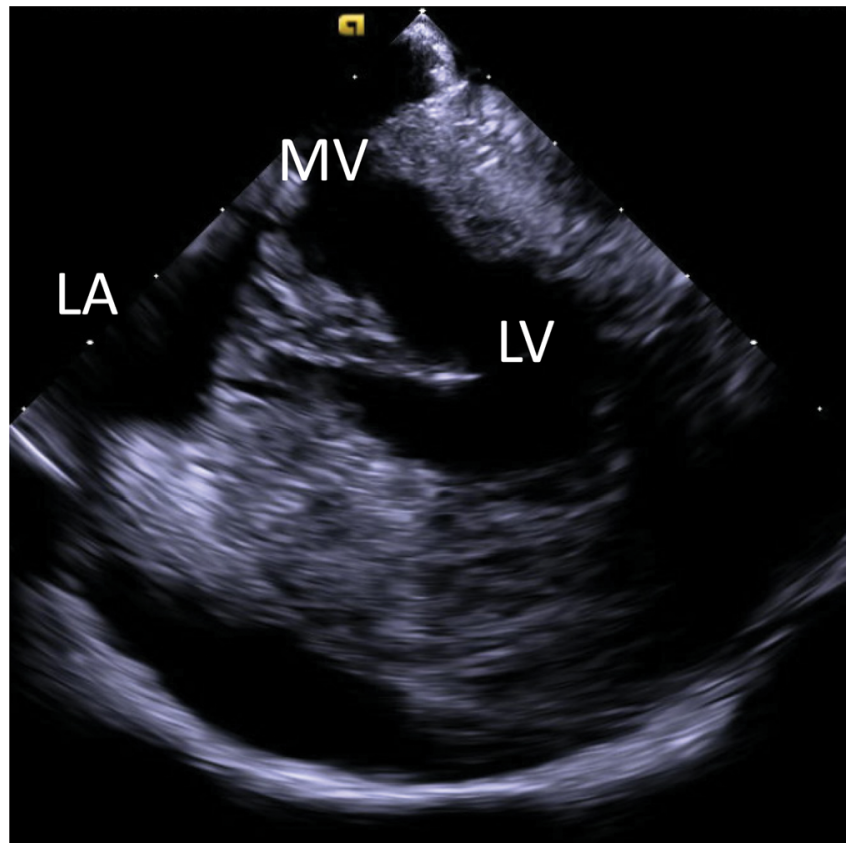

Figure 8. Transventricular long axis view. Posterior-tilted catheter is advanced into right ventricle. $L A=$ left atrium; LV = left ventricle; $M V=$ mitral valve; $R V=$ right ventricle. 
septum (Figure 10). During the puncture, the transseptal needle inserted into the transseptal sheath is directed to the fossa ovalis and carefully advanced. Successful puncture can be confirmed by contrast or agitated saline injection through the transseptal needle to the left atrium (Figure 11). The puncture site is tailored to the intended procedure. The superior-inferior position is evaluated in this long axis view, while the anterior-posterior position is evaluated by the short axis view.

\section{Atrial Septal Defect/Patent Foramen Ovale Closure}

Previous studies have shown that ICE provides excellent utility for percutaneous closure of ASD and PFO $[8,9]$. There is an increasing trend toward the use of ICE in the majority of these two procedures. Direct visualization may identify septum morphology (Figures 12, 13). The short axis view allows for evaluation of the anterior and posterior side of the rim, while the long axis view allows visualization of the superior and inferior side of the rim, as mentioned earlier. During the procedure, this view also allows visualization of guidewire insertion from the right to left atrium; wire positioning; optimal device sizing, positioning, and deployment; and presence of residual shunt (Figures 14, 15).

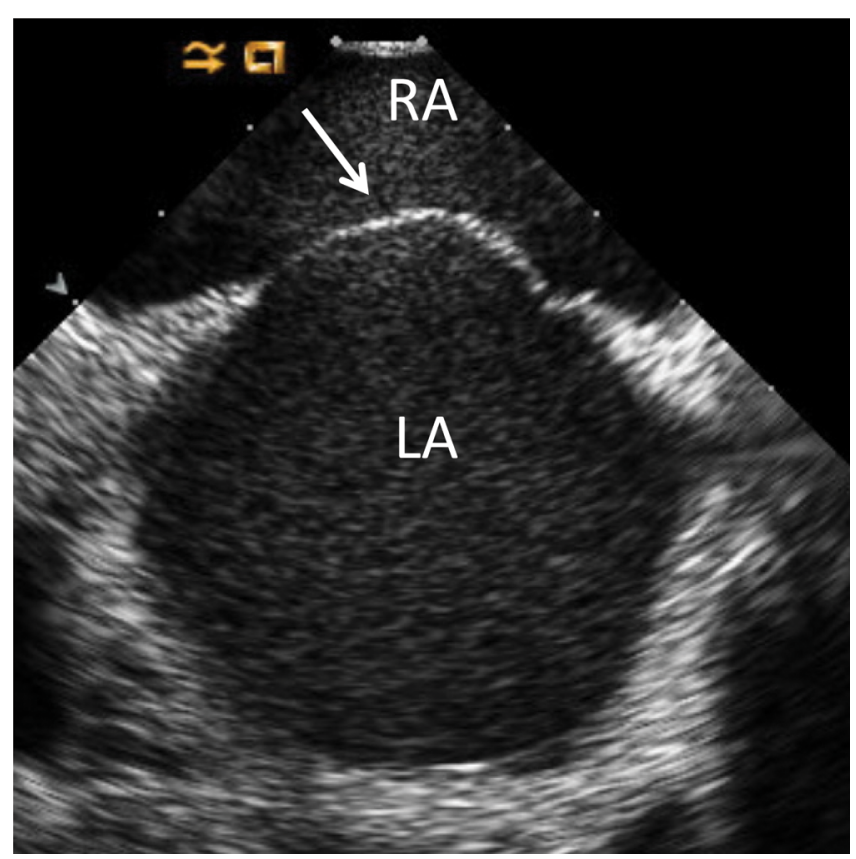

Figure 9. Long axis view. An atrial septal abnormality can be confirmed (arrow). LA, left atrium; RA, right atrium.

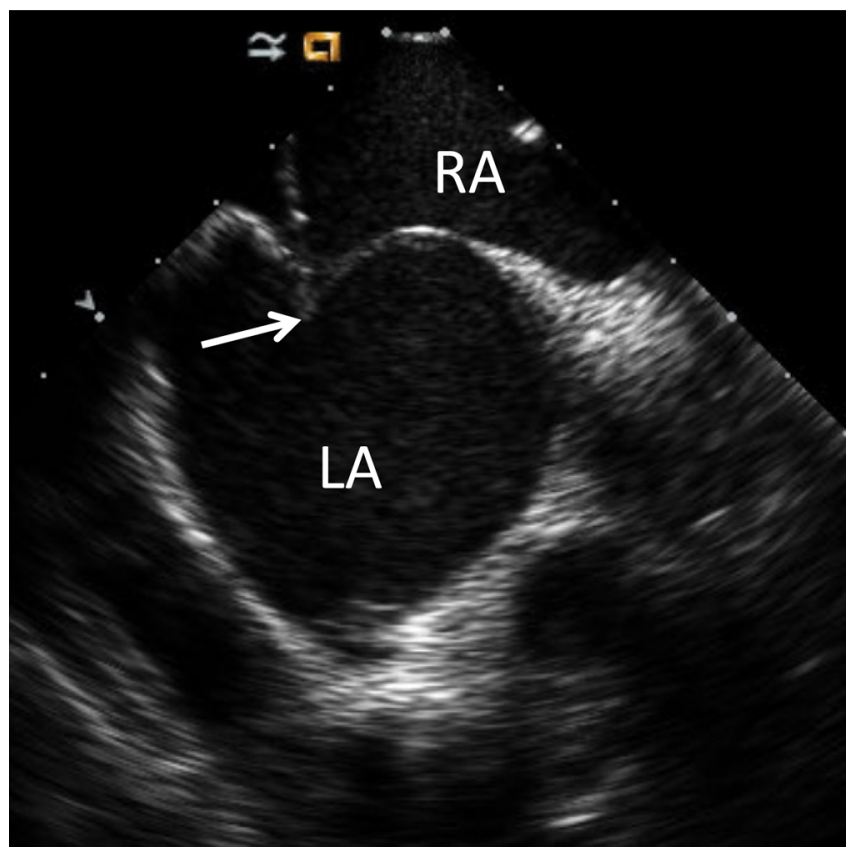

Figure 10. Long axis view. Tenting of the septum indicates stable contact of the needle with septum (arrow). LA = left atrium; $\mathrm{RA}=$ right atrium.

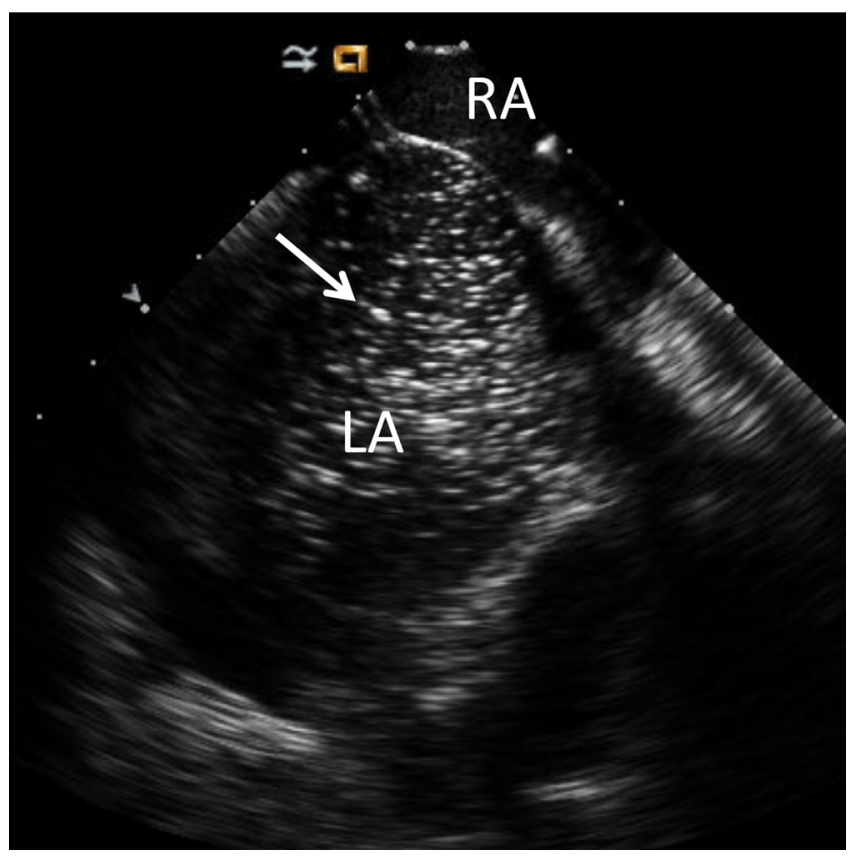

Figure 11. Long axis view. Successful puncture can be confirmed by contrast injection through the transseptal needle into left atrium (arrow). LA = left atrium; RA = right atrium. 


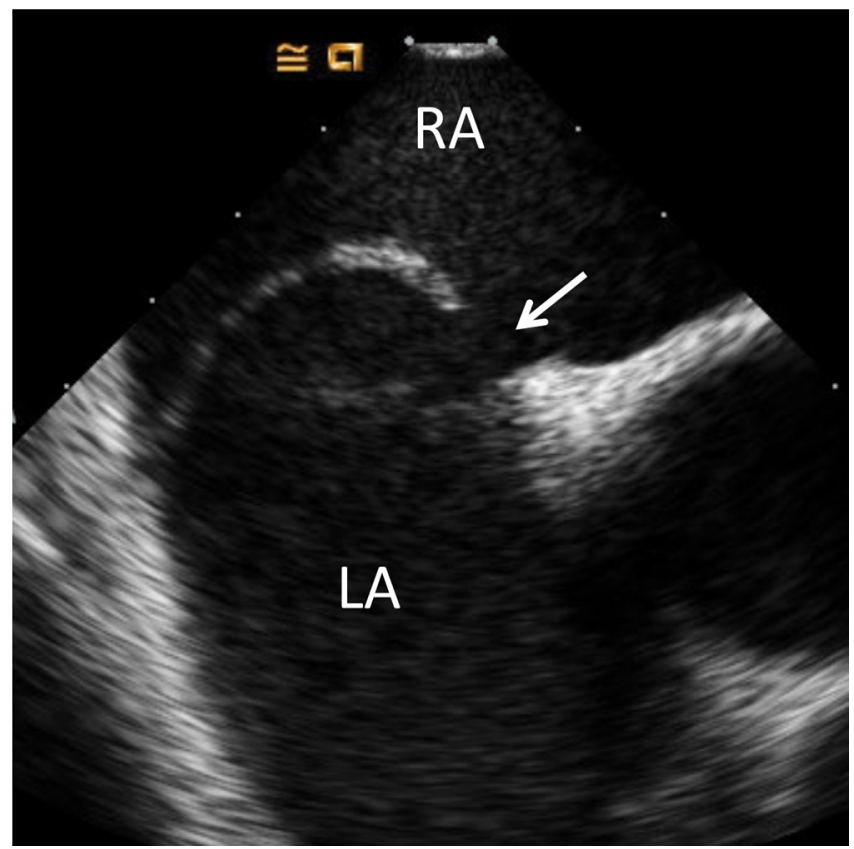

Figure 12. Long axis view. A secundum atrial septal defect can be seen (arrow). LA = left atrium; RA = right atrium.

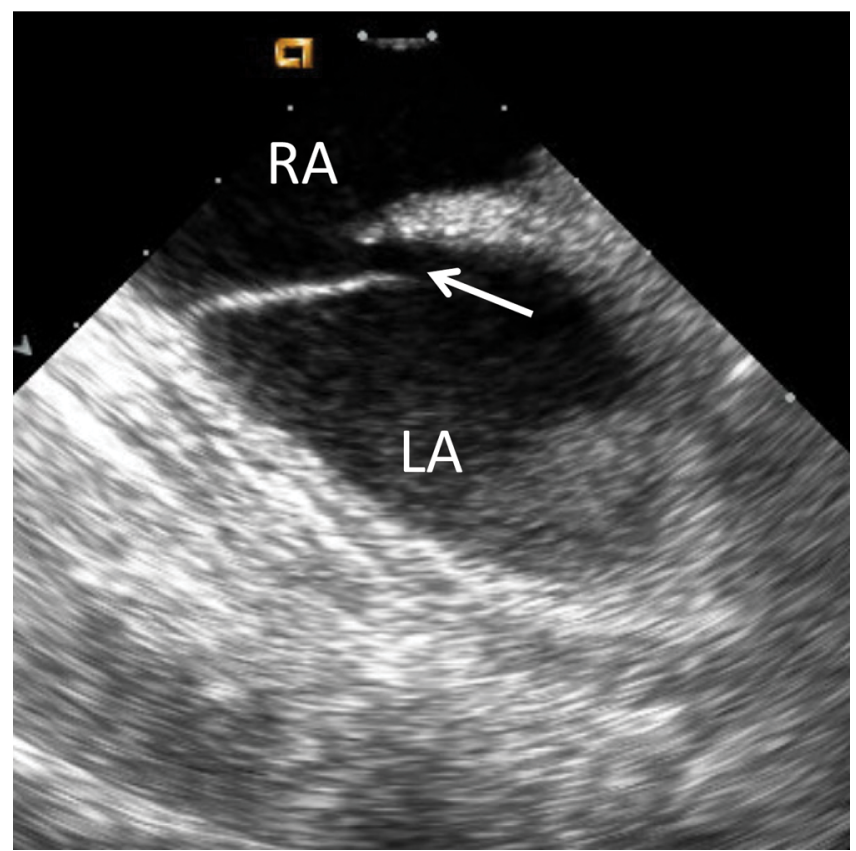

Figure 13. Long axis view. Patent foramen ovale can be seen (arrow). LA = left atrium; RA = right atrium.

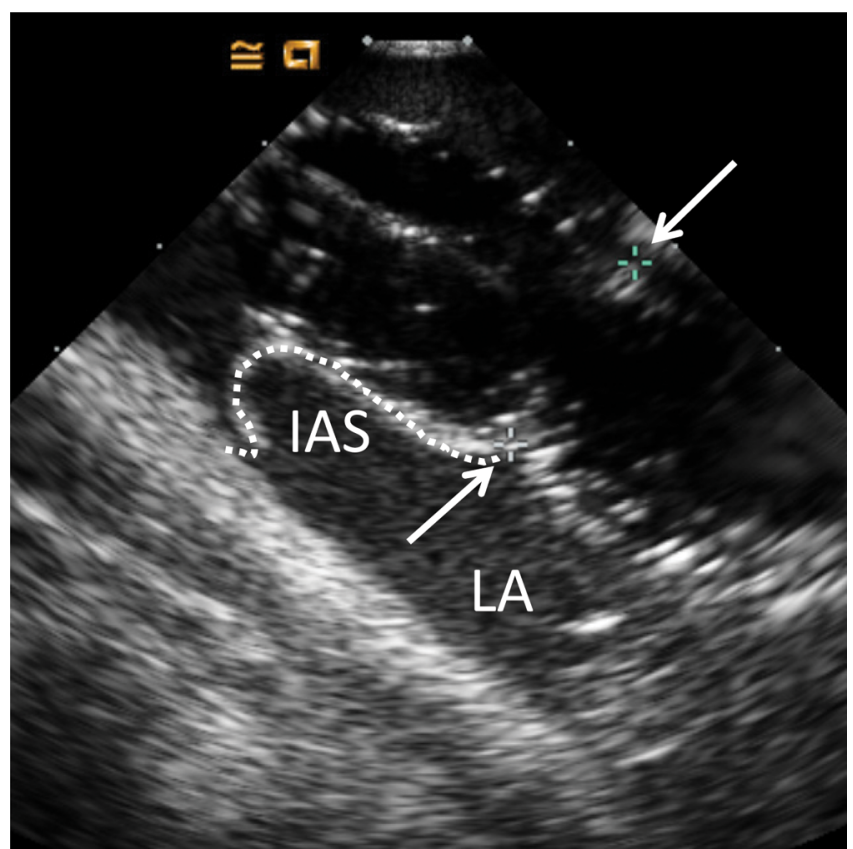

Figure 14. Balloon sizing before device deployment. Atrial septal defect is evaluated using sizing balloon (arrow). IAS = intra-atrial septum; LA = left atrium.

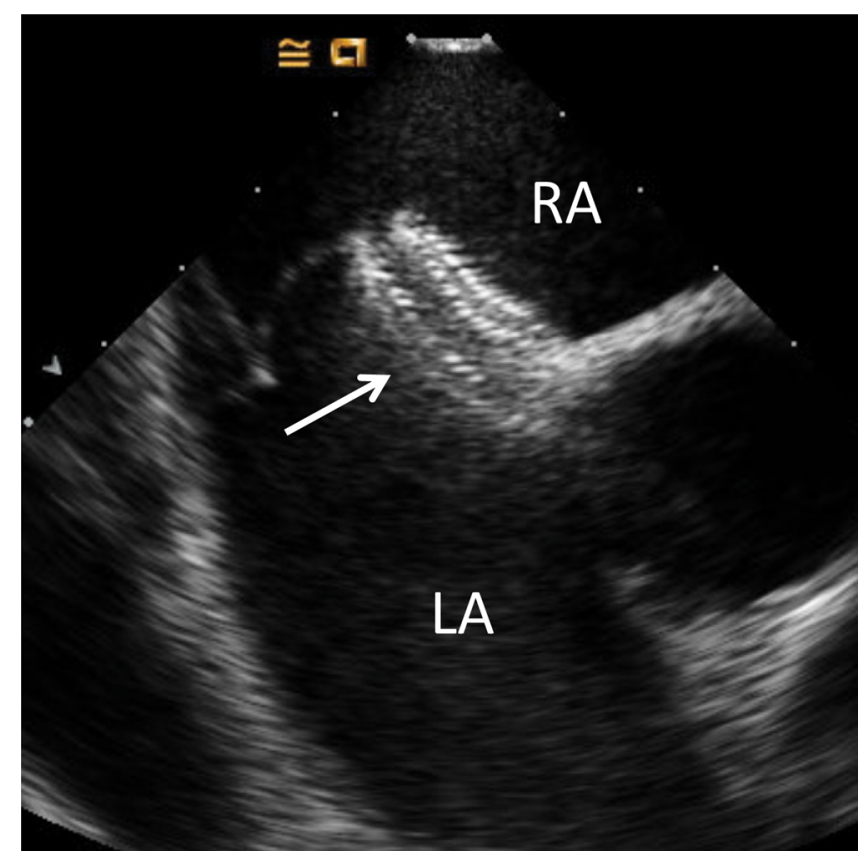

Figure 15. Atrial septal defect (ASD) closure device deployment. The ASD closure device is implanted (arrow). LA = left atrium; $\mathrm{RA}=$ right atrium. 


\section{Mitral Stenosis with Percutaneous Mitral Balloon Valvuloplasty}

Percutaneous mitral balloon valvuloplasty is a treatment of choice in patients with rheumatic mitral stenosis. ICE facilitates safer transseptal puncture, which is the riskiest part of this procedure. In addition, the long axis view can identify mitral apparatus deformity due to rheumatic disease, balloon positioning on the mitral valve, and the transmitral gradient after balloon dilation using Doppler (Figure 16).

\section{Aortic Stenosis with Transcatheter Aortic Valve Replacement}

A previous study compared imaging between ICE and TEE during TAVR and demonstrated the safety and feasibility of ICE-guided TAVR [11]. The long axis view (Figures 17,18) allows visualization of device positioning and deployment. The short axis view (Figure 7) is helpful to grade and evaluate paravalvular leak after device deployment. TEE remains the gold standard imaging modality for TAVR due to its multiplanar capabilities; however, ICE can be considered as an alternative in selected cases.

\section{Mitral Regurgitation with Transcatheter Mitral Valve Repair}

ICE may be useful during transcatheter mitral valve repair with the MitraClip (Abbott Vascular, Menlo Park, CA). As the mitral valve has a more complex morphology than the aortic valve, it needs a specific planar window in which both the A2 and P2 scallops of the mitral valve can be visualized (most frequent location for MitraClip placement), so the current generation of two-dimensional ICE has limited usage. However, if there is difficulty visualizing pathology with TEE because of shadowing from the prior surgical ring or severe mitral annular calcification (Figure 19), adjunctive ICE has been helpful in the authors' experience. To adequately visualize insertion of both the anterior and posterior leaflets into the MitraClip, the $8 \mathrm{~F} \mathrm{ICE}$

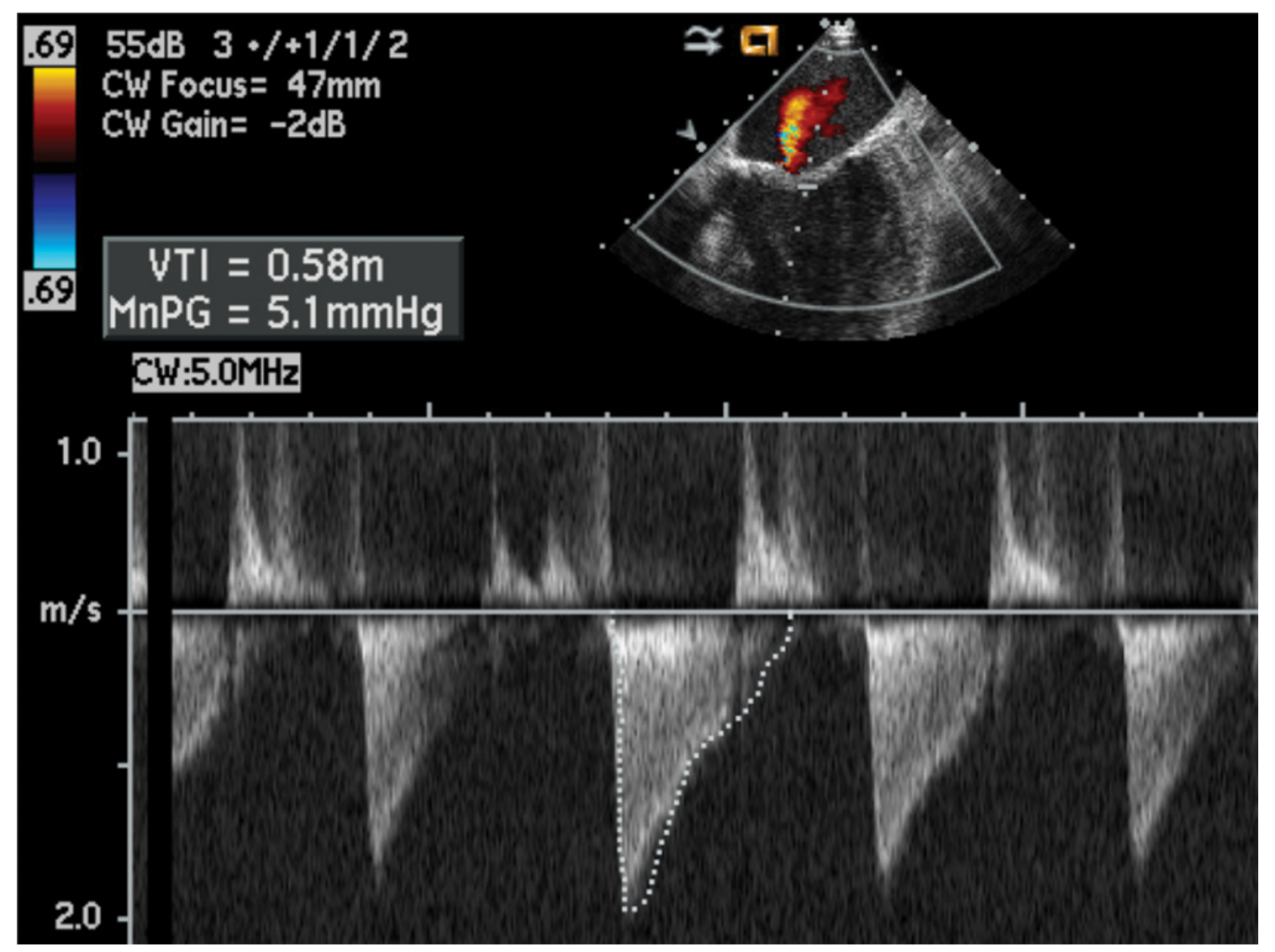

Figure 16. Transmitral gradient obtained by intracardiac echocardiography. Color Doppler identifies the transmitral gradient. 

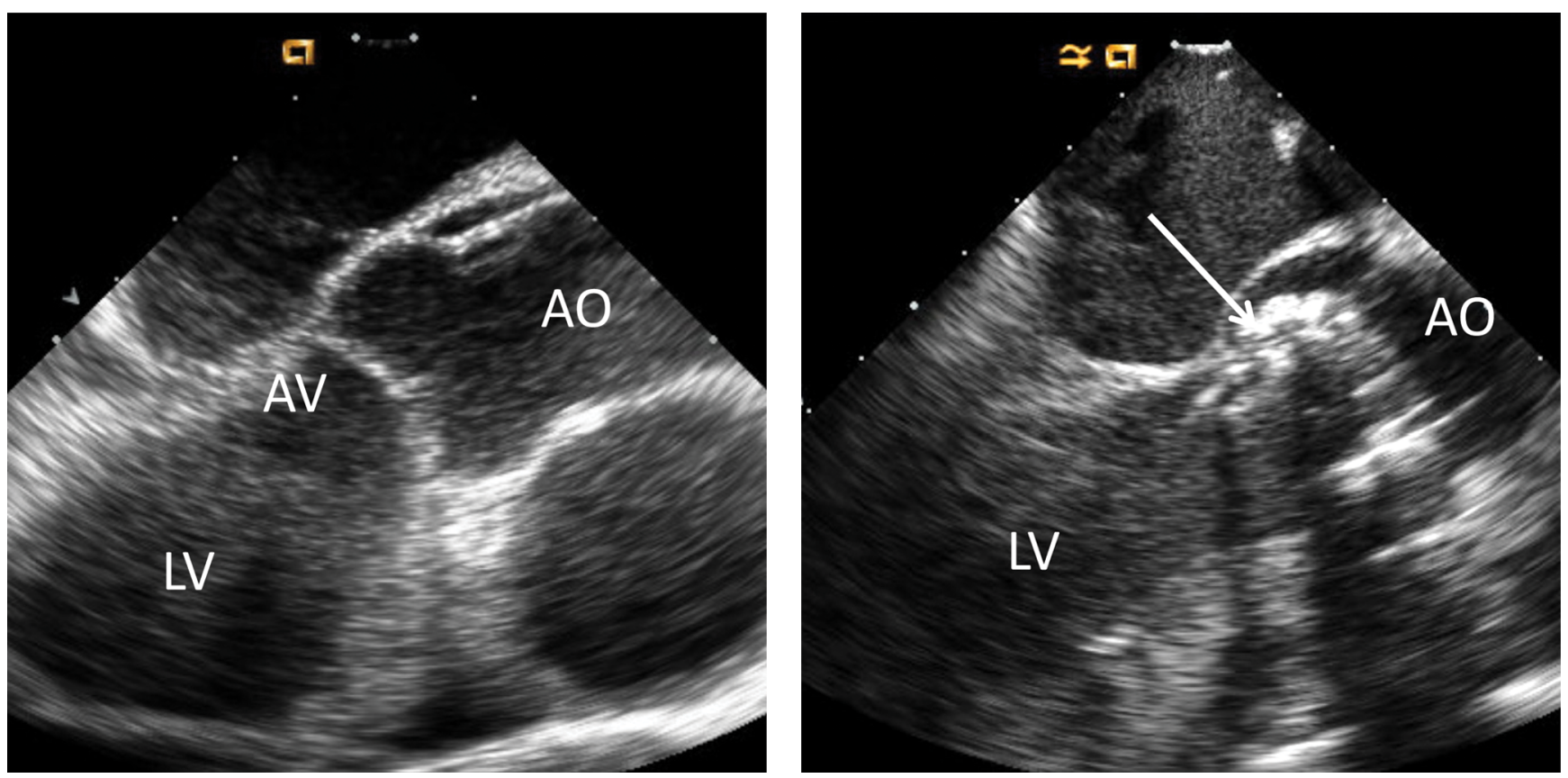

Figures 17 and 18. Long axis view in transcatheter aortic valve replacement. Long axis view from high right atrium allows the visualization of ascending aorta, aortic valve, and left ventricle; outflow tract. A bioprosthetic valve is implanted percutaneously (arrow). $\mathrm{AO}=$ ascending aorta; $\mathrm{AV}=$ aortic valve; $\mathrm{LV}=$ left ventricle; $\mathrm{RA}=$ right atrium.

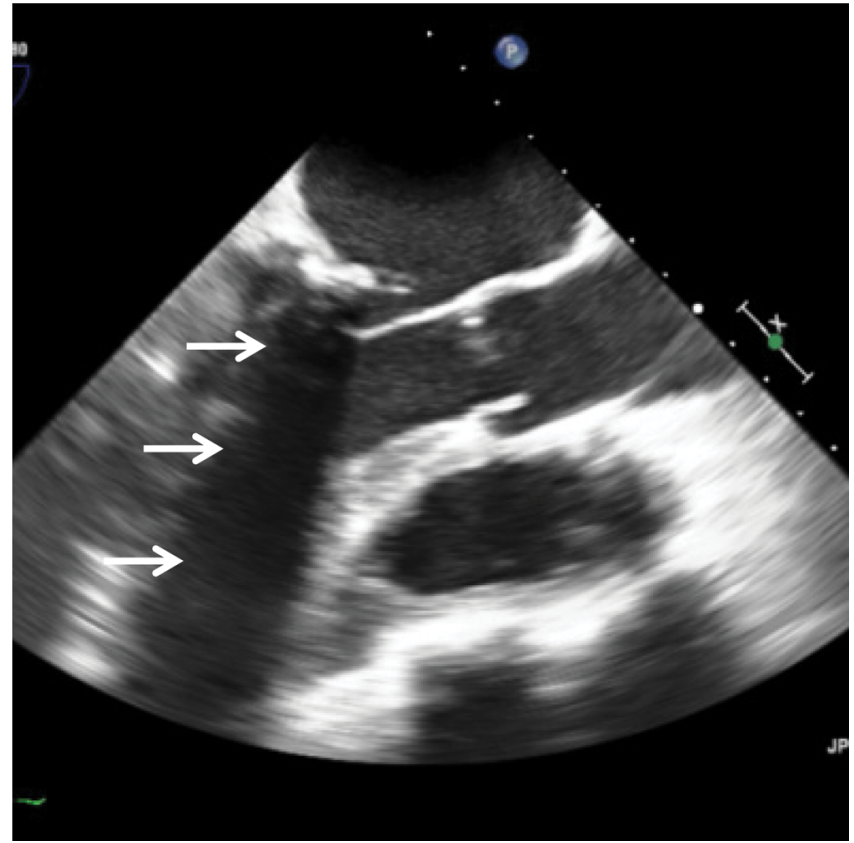

Figure 19. Imaging obtained by transesophageal echocardiography. Posterior leaflet is difficult to see because of shadowing from the prior surgical ring (arrow).

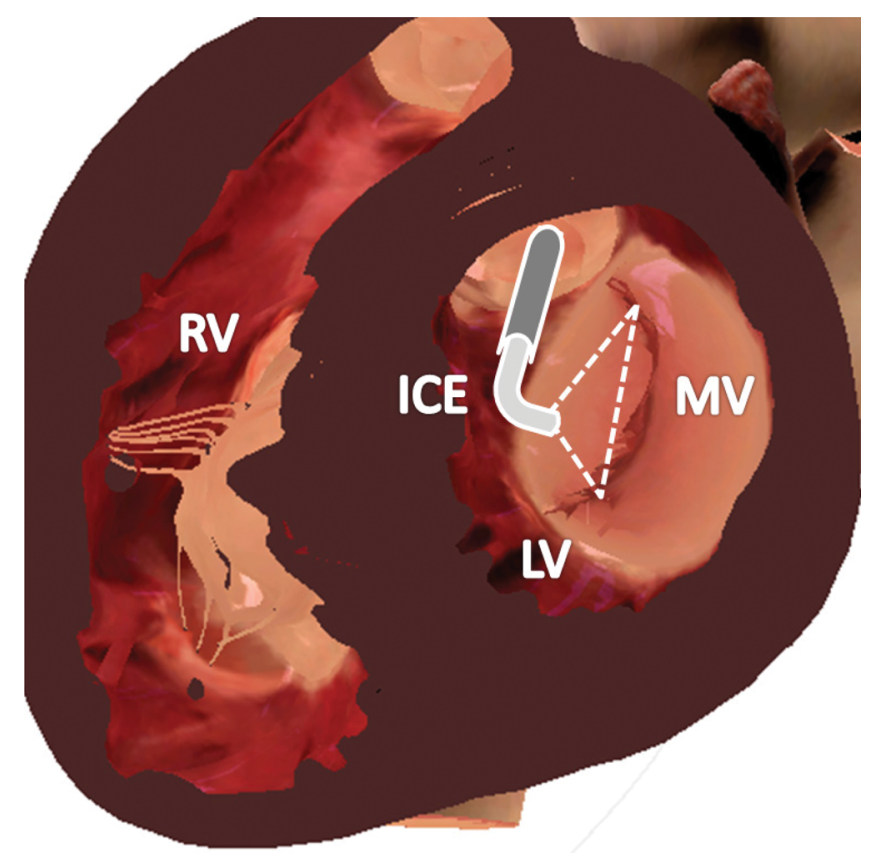

Figure 20. Retrograde and transarterial introduction of the 8-French intracardiac echocardiography catheter into the left ventricle. $L V=$ left ventricle; $M V=$ mitral valve; $R V=$ right ventricle. 
catheter is introduced retrogradely and transarterially into the left ventricle and flexed to image across the mitral valve (Figure 20). The double-orifice mitral valve created by the MitraClip can be visualized by
ICE (Figure 21). Notably, in transcatheter mitral valve repair, three-dimensional ICE remains of limited utility due to its relatively narrow sector width and inadequate resolution (Figure 22).
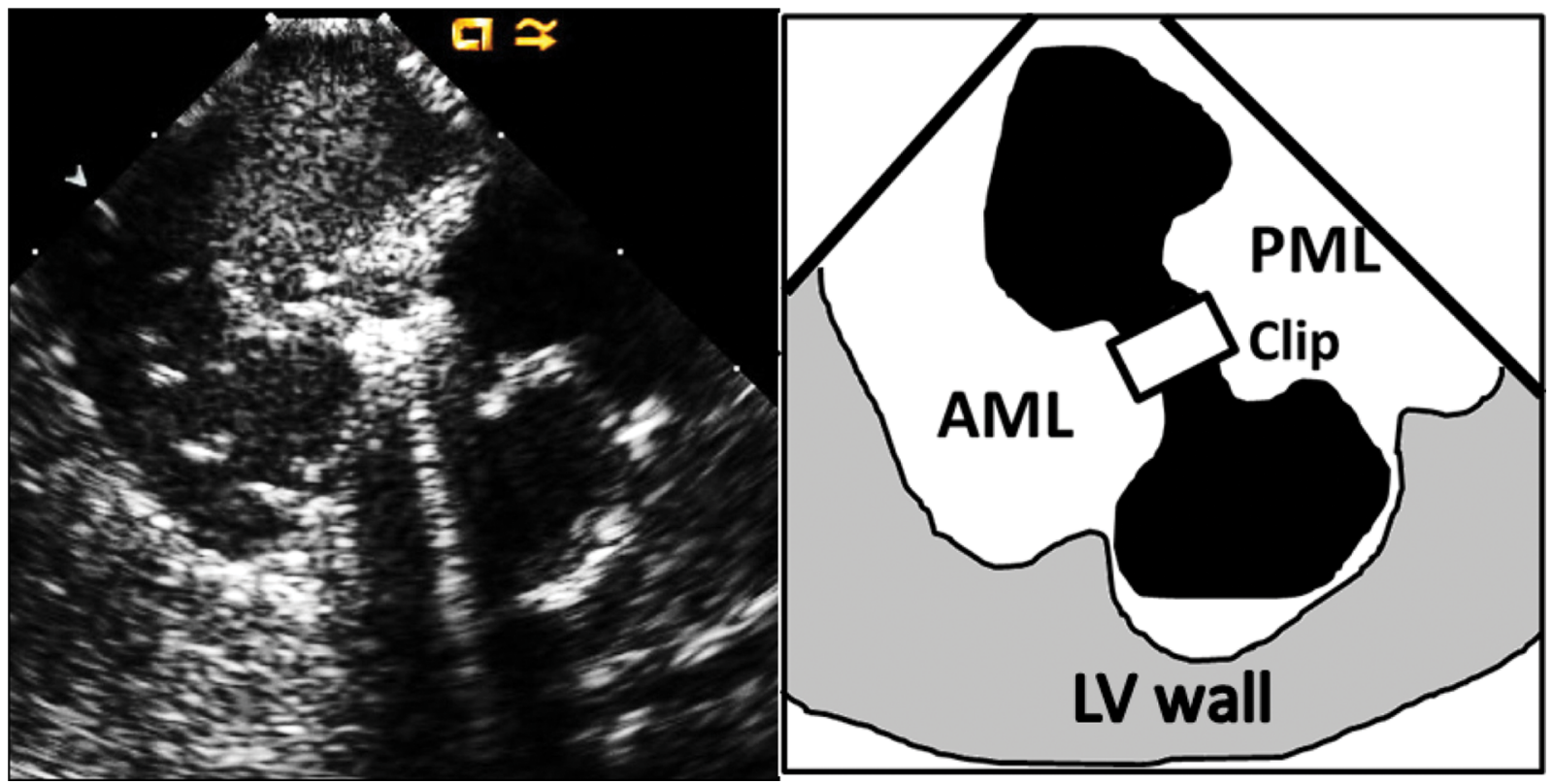

Figure 21. The left ventricle short axis view obtained by intracardiac echocardiography (ICE). ICE can demonstrate appropriate insertion of both leaflets into the MitraClip creating the double-orifice mitral valve. $A M L=$ anterior mitral leaflet; $L V=l e f t$ ventricle; $P M L=$ posterior mitral leaflet.

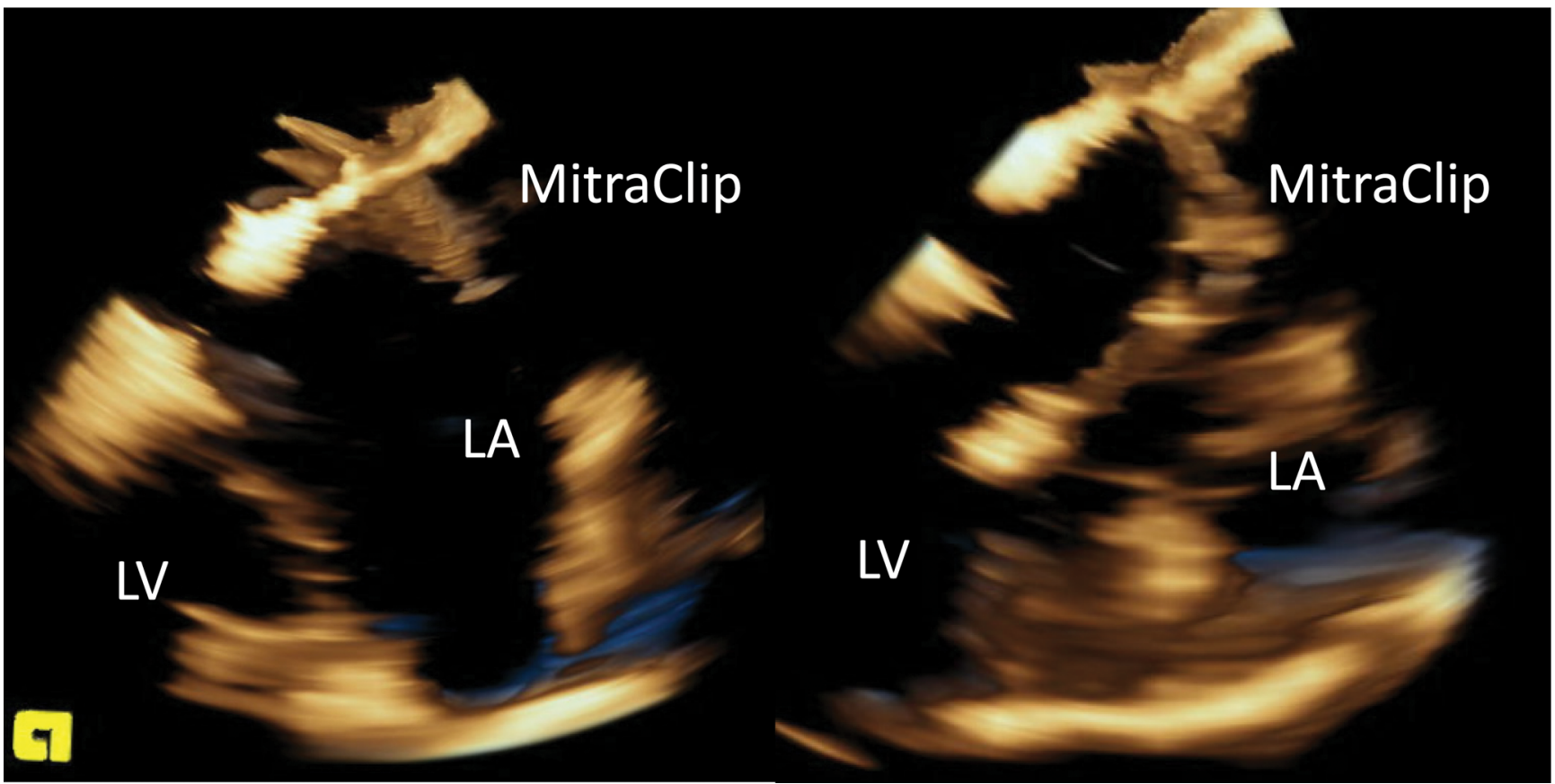

Figure 22. Three-dimensional intracardiac echoc-ardiography (ICE) imaging. Three-dimensional ICE guides the MitraClip delivery system. $L A$, = left atrium; LV = left ventricle. 


\section{Left Atrial Appendage Closure}

The current generation of single-plane ICE catheter that creates imaging from the right atrium is rarely sufficient to fully visualize the complicated anatomy of the left atrial appendage [16], and there have been only case reports describing successful appendage closures with ICE monitoring $[6,17]$. Future ICE iterations with multiplanar capability would allow more accurate measurement of the ostial diameter, which is not circular, and postprocedural color Doppler to check for device leaks.

\section{Conclusions}

ICE has been used to a variable degree in structural heart interventions. While it has an excellent clinical role for guiding ASD/PFO closure, technologic limitations hamper its application during other structural interventions. Multiplanar imaging and wide-sector three-dimensional imaging are required to improve ICE utilization during transcatheter interventional procedures.

\section{Conflict of Interest}

The authors have no conflict of interest relevant to this publication.

\section{References}

1. Kort S. Intracardiac echocardiography: evolution, recent advances, and current applications. J Am Soc Echocardiogr. 2006;19:11921201. DOI: 10.1016/j.echo.2006.04.033

2. Hijazi ZM, Shivkumar K, Sahn DJ. Intracardiac echocardiography during interventional and electrophysiological cardiac catheterization. Circulation. 2009;119:587-596. DOI: 10.1161/CIRCULATIONAHA.107.753046

3. Ali S, George LK, Das P, Koshy SK. Intracardiac echocardiography: clinical utility and application. Echocardiography. 2011;28:582-590. DOI: 10.1111/j.15408175.2011.01395.x

4. Baran J, Stec S, Pilichowska-Paszkiet E, Zaborska B, Sikora-Frąc M, Kryński T, et al. Intracardiac echocardiography for detection of thrombus in the left atrial appendage: comparison with transesophageal echocardiography in patients undergoing ablation for atrial fibrillation: the Action-Ice I Study. Circ Arrhythm Electrophysiol. 2013;6:10741081. DOI: 10.1161/CIRCEP.113.000504

5. Reddy VY, Neuzil P, Ruskin JN. Intracardiac echocardiographic imaging of the left atrial appendage. Heart Rhythm. 2005;2:12721273. DOI: $10.1016 /$ j.hrthm.2005.06.027

6. Fassini G, Dello Russo A, Conti S, Tondo C. An alternative transseptal intracardiac echocardiography strategy to guide left atrial appendage closure: the first described case. J Cardiovasc Electrophysiol. 2014;25:12691271. DOI: $10.1111 /$ jce. 12480

7. Bidart C, Vaseghi M, Cesario DA, Mahajan A, Fujimura O, Boyle NG, et al. Radiofrequency current delivery via transseptal needle to facilitate septal puncture. Heart Rhythm. 2007;4:1573-1576. DOI: 10.1016/j. hrthm.2007.07.008

8. Bartel T, Konorza T, Arjumand J, Ebradlidze T, Eggebrecht H, Caspari G, et al. Intracardiac echocardiography is superior to conventional monitoring for guiding device closure of interatrial communications. Circulation. 2003;107:795-797. DOI: 10.1161/01.CIR.0000057547.00909.1C

9. Earing MG, Cabalka AK, Seward JB, Bruce CJ, Reeder GS, Hagler DJ. Intracardiac echocardiographic guidance during transcatheter device closure of atrial septal defect and patent foramen ovale. Mayo Clin Proc. 2004;79:24-34. DOI: 10.4065/79.1.24

10. Green NE, Hansgen AR, Carroll JD. Initial clinical experience with intracardiac echocardiography in guiding balloon mitral valvuloplasty: technique, safety, utility, and limitations. Catheter Cardiovasc Interv. 2004;63:385-394. DOI: 10.1002/ccd.20177

11. Bartel T, Bonaros N, Müller L, Friedrich G, Grimm M, Velik-Salchner C, et al. Intracardiac echocardiography: a new guiding tool for transcatheter aortic valve replacement. J Am Soc Echocardiogr. 2011;24:966-975. DOI: 10.1016/j.echo.2011.04.009

12. Oishi $Y$, Okamoto $M$, Sueda $T$, Hashimoto M, Karakawa S, Akita T, et al. Cardiac tumor biopsy under the guidance of intracardiac echocardiography. Jpn Circ J. 2000;64:638640. DOI: $10.1253 /$ jcj.64.638

13. Cohen TJ, Juang G. Utility of intracardiac echocardiography to facilitate transvenous coronary sinus lead placement for biventricular cardioverter-defibrillator implantation. J Invasive Cardiol. 2003;15:685-686.

14. Cao QL, Zabal C, Koenig P, Sandhu S, Hijazi ZM. Initial clinical experience with intracardiac echocardiography in guiding transcatheter closure of perimembranous ventricular septal defects: feasibility and comparison with transesophageal echocardiography. Catheter Cardiovasc Interv. 2005;66:258-267. DOI: 10.1002/ccd.20463

15. Pedone C, Vijayakumar $M$, Ligthart JM, Valgimigli $M$, Biagini $E$, De Jong $N$, et al. Intracardiac echocardiography guidance during percutaneous transluminal septal myocardial ablation in patients with obstructive hypertrophic cardiomyopathy. Int J Cardiovasc Intervent. 2005;7:134-137. DOI: $10.1080 / 14628840500280575$

16. Desimone CV, Asirvatham SJ. ICE Imaging of the left atrial appendage. J Cardiovasc Electrophysiol. 2014;25:1272-1274. DOI: 10.1111/jce.12536

17. MacDonald ST, Newton JD, Ormerod OJ. Intracardiac echocardiography off piste? Closure of the left atrial appendage using ICE and local anesthesia. Catheter Cardiovasc Interv. 2011;77:124-127. DOI: 10.1002/ ccd. 22652

Cite this article as: Saji M, Lim DS. Use of Intracardiac Echocardiography for Congenital and Structural Heart Disease Interventions. Structural Heart Disease. 2016;2(4):108-117. DOI: http://dx.doi. org/10.12945/j.jshd.2016.006.15 